\title{
Tumor vasoproliferativo idiopático da retina associado à hemorragia vítrea recidivante - Relato de caso
}

\author{
Idiopathic vasoproliferative tumor of the retina associated with recurring vitreous \\ hemorrhage-Case report
}

Pedro D. Serracarbassa ${ }^{1}$

Eduardo Cunha de Souza ${ }^{2}$

\section{RESUMO}

Objetivo: Os autores apresentam dois casos raros e ilustrativos de tumor vasoproliferativo, idiopático presumido da retina associado à hemorragia vítrea recidivante, em duas pacientes jovens e sadias. Método: Dois olhos de duas pacientes foram avaliados por meio do exame de fundoscopia, retinografia, angiofluoresceinografia e ultra-sonografia. Resultados: Nos dois casos relatados, o tumor vascular retiniano foi unilateral, solitário e apresentou evidências ultra-sonográficas de adesão vítrea. São discutidos os diagnósticos diferenciais da doença e alternativas de tratamento com crioterapia, fotocoagulação e vitrectomia. Conclusão: Os autores ressaltam a importância do reconhecimento do tumor vasoproliferativo idiopático da retina nos casos de hemorragia vítrea recidivante.

Descritores: Hemangioma/patologia; Neoplasias da retina/patologia; Vasos retinianos/ patologia; Neovascularização patológica; Hemorragia vítrea; Relato de caso

\section{INTRODUÇ̃̃O}

Os tumores vasoproliferativos idiopáticos da retina, também chamados de hemangiomas retinianos adquiridos ${ }^{(1)}$ ou tumores "hemangioma-like" da retina $^{(2)}$, são tumores relativamente raros, inicialmente descritos por Shields et al. ${ }^{(1)}$ em 1983. Tratam-se de massas vasculares periféricas, unilaterais, solitárias, de coloração rósea ou branca, acometendo a retina sensorial. Outros achados incluem: exsudação ao redor da lesão, hemorragias retinianas, descolamento exsudativo da retina e hemorragia vítrea. $\mathrm{O}$ aspecto angiofluoresceinográfico mostra um enchimento precoce da trama vascular com áreas de extravasamento tardio do contraste, sem áreas de oclusão capilar. Observam-se também vasos nutridores e de drenagem, não dilatados, direcionados para o tumor. Estas lesões foram posteriormente classificadas como tumores vasoproliferativos primários e secundários de acordo com sua etiologia. No presente estudo, relatam-se dois casos de um provável tumor vasoproliferativo idiopático da retina associado à hemorragia vítrea e discutem-se aspectos do seu diagnóstico diferencial e tratamento.

\section{RELATO DE CASO}

\section{Caso 1}

A. L. T, 32 anos, sexo feminino, branca, natural e procedente de São Paulo. A paciente apresentava queixa de perda súbita de visão do olho 
direito (OD) há 15 dias. Foi diagnosticada e tratada em outro serviço como "uveíte a esclarecer" com corticoterapia oral sem melhora. Quando procurou nosso serviço, apresentava os seguintes dados no exame oftalmológico: acuidade visual de conta dedos a 1 metro no OD e 20/20 no OE, segmento anterior sem anormalidades e pressão intra-ocular de $13 \mathrm{mmHg}$ em ambos os olhos (AO). No exame fundoscópico encontrouse hemorragia vítrea e lesão periférica temporal inferior de coloração rósea, elevada e vascularizada (Figura 1). No exame angiofluoresceinográfico observou-se um enchimento precoce da lesão com extravasamento tardio do contraste. Não foram observadas áreas de má-perfusão capilar (Figura 2).

No exame ultra-sonográfico observou-se imagem sugestiva de hemorragia na cavidade vítrea e descolamento parcial do vítreo posterior, estando este aderido apenas no local da tumoração retiniana (Figura 3). Os antecedentes pessoais e familiares da paciente eram normais, não havendo, portanto, história familiar de tumores oculares e do sistema nervoso central. Os exames subsidiários para: diabetes melitus, hemoglobinopatias, síndromes de hiperviscosidade, vasculites do tipo lupus, sarcoidose e hemangioblastoma cerebelar, foram negativos. O tratamento realizado consistiu da retirada da corticoterapia oral e observação.

Trinta dias após, houve diminuição da hemorragia vítrea e melhora da acuidade visual do OD para 20/30, quando então se optou por fotocoagular a lesão vascular. Vinte dias após o tratamento com fotocoagulação, a paciente retornou com nova diminuição súbita da acuidade visual do OD para movimento de mãos devido à hemorragia vítrea. Optou-se então pela vitrectomia e aplicação de endolaser sobre a lesão vascular. A paciente foi acompanhada até o nonagésimo dia de pós-operatório apresentando, no último dia de seguimento, acuidade visual de 20/30 no OD e lesão vascular de aspecto esbranquiçado, circundada por áreas de cicatrizes de coriorretinite induzidas pela fotocoagulação.

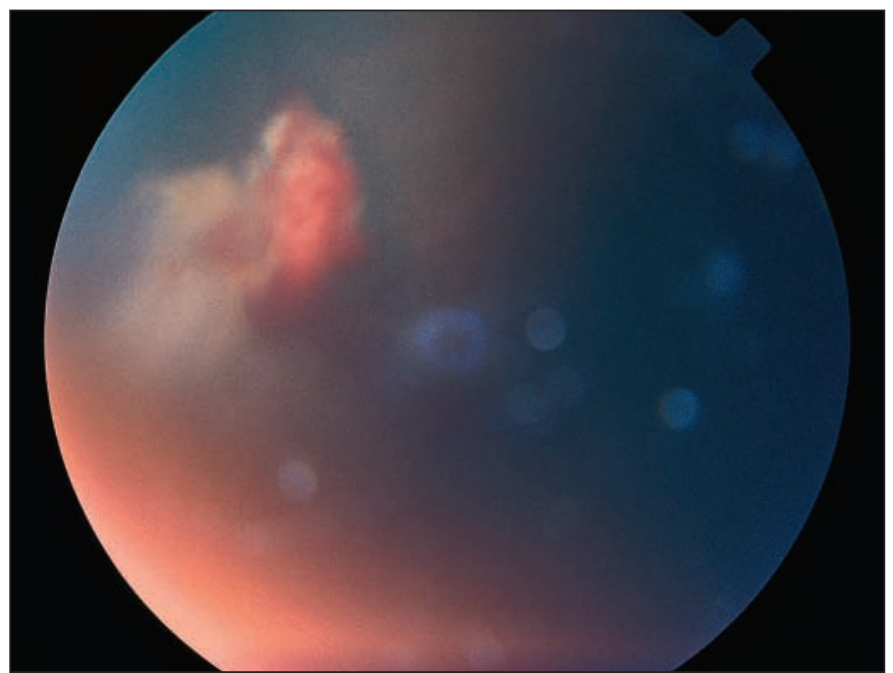

Figura 1 - Retinografia do olho direito (caso 1). Observa-se lesão periférica, elevada, de coloração rósea e vascularizada

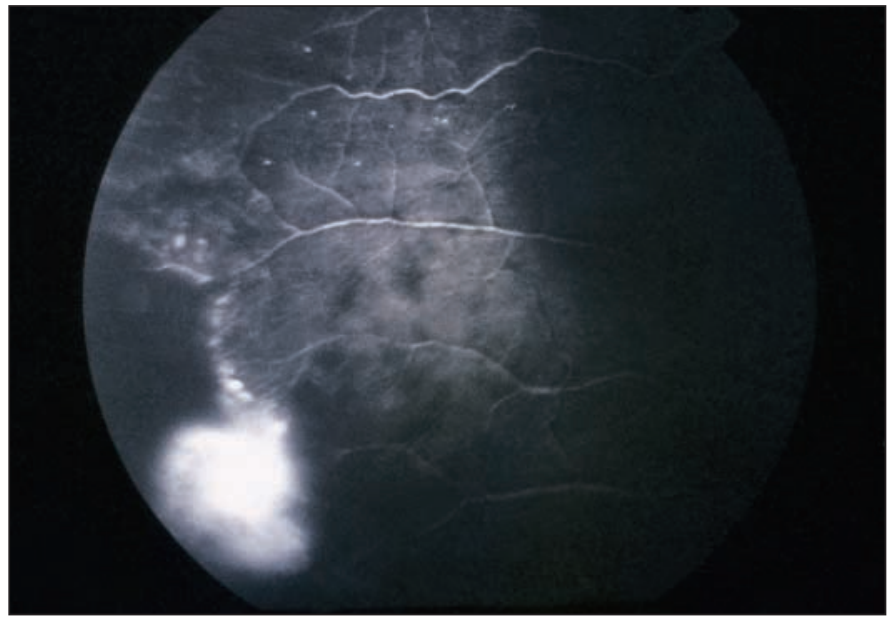

Figura 2 - Angiofluoresceinografia do olho direito (caso 1). Observa-se lesão com extravasamento tardio do contraste

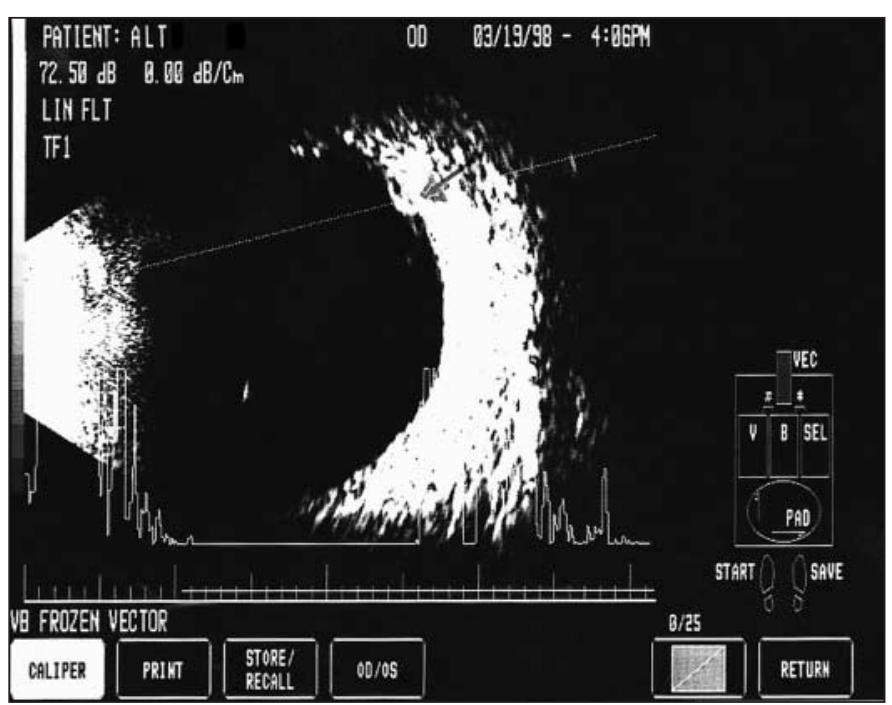

Figura 3 - Ultra-sonografia do olho direito (caso 1). Observa-se tumoração periférica aderida à hialóide posterior

\section{Caso 2}

J. C. S., 38 anos, sexo feminino, natural e procedente da Bahia. Apresentava queixa de diminuição súbita da acuidade visual no OD há 1 semana. Os antecedentes oculares, familiares e pessoais mostravam-se normais. No exame clínico, foi encontrada acuidade visual no OD de 20/400 e no OE de 20/20. A biomicroscopia apresentava-se sem anormalidades e a pressão intra-ocular era de $12 \mathrm{mmHg}$ no olho direito e $14 \mathrm{mmHg}$ no olho esquerdo. No exame fundoscópico, observou-se hemorragia vítrea que impedia uma observação detalhada do pólo posterior. A paciente foi submetida a exame ultra-sonográfico onde se evidenciou a presença de lesão elevada, sobre a retina, localizada no quadrante temporal inferior e aderida à hialóide posterior (Figura 4).

Foram solicitados exames complementares para: diabetes melitus, hemoglobinopatias, síndromes de hiperviscosidade, 


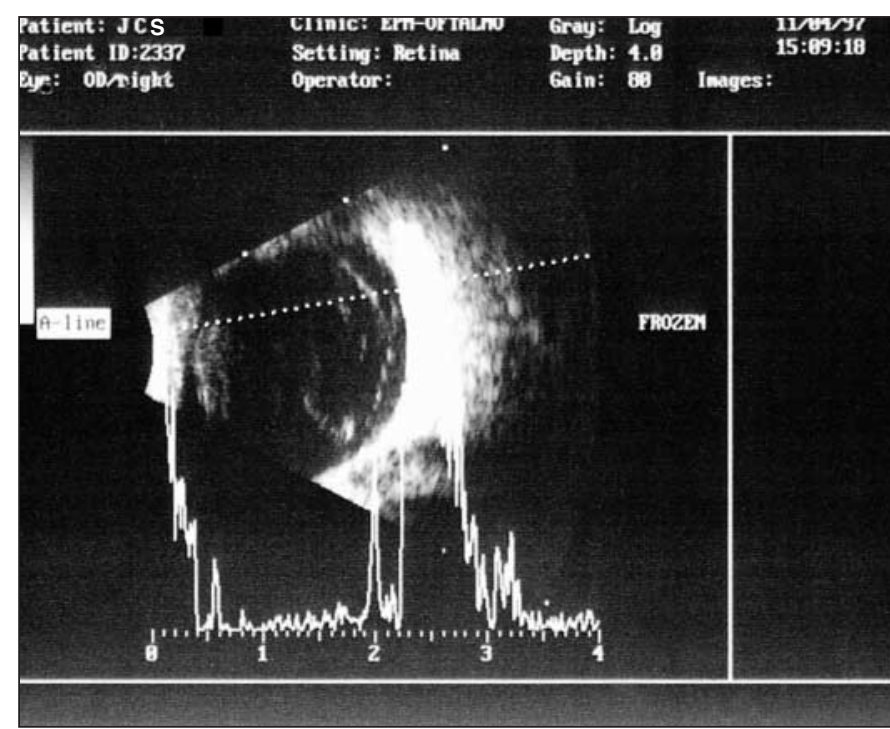

Figura 4 - Ultra-sonografia do olho direito (caso 2). Observa-se descolamento parcial do vítreo posterior

vasculites e anomalias vasculares do S. N. C. A conduta seguida neste caso foi a observação. Quinze dias após a consulta inicial, a hemorragia vítrea apresentou sinais de reabsorção, sendo então possível observar o segmento posterior. Observou-se no quadrante temporal inferior um tumor elevado, de coloração avermelhada, medindo ao redor de 1 diâmetro papilar, com bordas bem delimitadas (Figura 5).

A paciente foi submetida a tratamento com fotocoagulação com laser de argônio, inicialmente ao redor da lesão, e posteriormente no próprio tumor.

Quarenta dias após o tratamento, novo episódio de hemorragia vítrea foi diagnosticado e a conduta foi novamente conservadora. A hemorragia apresentou sinais de melhora trinta dias após e nova aplicação de laser de argônio foi realizada sobre a tumoração.

A paciente foi acompanhada durante seis meses após a segunda aplicação de laser, sendo que, o último exame do segmento posterior mostrou uma lesão esbranquiçada circundada por cicatrizes hipertróficas de epitélio pigmentado da retina (marcas de fotocoagulação), sem sinais de hemorragia vítrea.

\section{DISCUSSÃO}

Os tumores vasoproliferativos idiopáticos da retina apresentam similaridades e diferenças em relação aos hemangiomas capilares da retina observados na doença de Von Hippel ${ }^{(3)}$. O aspecto clínico e angiofluoresceinográfico das lesões e a ausência de história familiar e sistêmica sugerem que estas lesões vasculares benignas adquiridas sejam uma forma variante dos hemangiomas capilares da retina ${ }^{(1)}$.

São descritos na literatura pacientes com massas vasculares semelhantes àquelas relatadas como formas adquiridas do hemangioma ${ }^{(1-2)}$. Analisando as características dos tumores

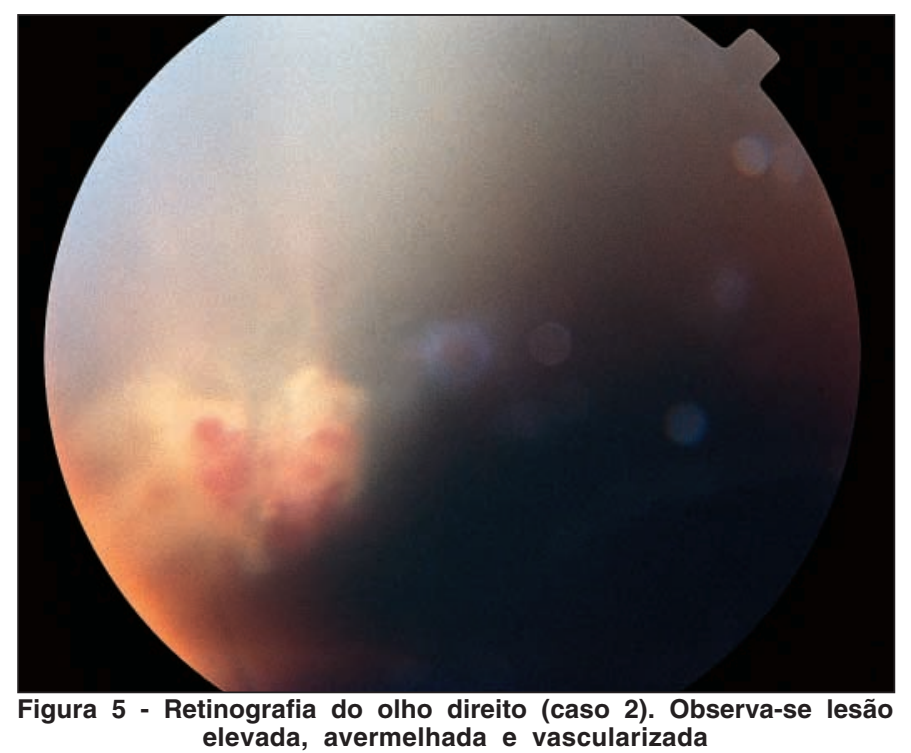

vasoproliferativos idiopáticos e dos hemangiomas capilares da doença de Von Hippel, observa-se que os primeiros apresentavam: idade de aparecimento mais avançada, história familiar negativa, ausência de lesões similares na família, ausência de acometimento sistêmico, falta de lesões sistêmicas na tomografia computadorizada, tumor unilateral, solitário e vasos nutridores não tortuosos. Concluíram, portanto, que os tumores vasoproliferativos idiopáticos seriam uma variante adquirida dos hemangiomas capilares, e representariam um tumor vascular benigno, necessitando, porém, de confirmação histopatológica.

Os relatos deste estudo apresentam as características clínicas e angiofluoresceinográficas citadas pelos autores acima. A ausência de história familiar e acometimento sistêmico sugere um provável quadro de tumor vasoproliferativo idiopático da retina. Entre as causas vasculares de tumores proliferativos, citamos: a retinopatia da prematuridade ${ }^{(4)}$, retinopatia diabética, anemia falciforme e outras retinopatias proliferativas $^{(5)}$. Estas doenças apresentam quadro de oclusão capilar no exame de angiofluoresceinográfico, fato este não observado nas descrições acima relatadas e no presente estudo.

A doença de Coats ${ }^{(6)}$ é um diagnóstico diferencial importante, porém as lesões apresentam-se menores, estão presentes desde o nascimento e tendem a exsudar com freqüência. Os tumores vasoproliferativos podem ser secundários a doenças pré-existentes como: pars planite, retinose pigmentar, toxoplasmose, toxocaríase, coloboma e coriorretinopatia traumática $^{(7)}$. O prognóstico visual destes tumores é variável.

É descrita perda significante da acuidade visual em 4 de 5 pacientes portadores do hemangioma adquirido ${ }^{(2)}$. Em estudo retrospectivo, foram avaliados 129 tumores vasoproliferativos em 113 olhos de 103 pacientes $^{(7)}$. As complicações associadas à diminuição da acuidade visual foram: hemorragia vítrea, exsudação intra-retiniana, descolamento exsudativo da retina, fibrose macular pré-retiniana e edema macular. Dos 113 olhos estudados, $24(21 \%)$ apresentaram episódios de hemorragia vítrea. 
No presente estudo são descritos dois casos de hemorragia vítrea recidivante como complicação destes tumores. $\mathrm{Na}$ análise do ultra-som, observamos um descolamento do vítreo posterior parcialmente aderido ao tumor e sinais de hemorragia vítrea. Acredita-se que a origem destas hemorragias tenha sido decorrente da tração vítrea exercida sobre o tumor, levando à ruptura dos vasos superficiais.

Devido ao grande número de complicações associadas aos tumores vasoproliferativos da retina, com perda visual significativa, recomenda-se que estes sejam tratados no momento do diagnóstico. O tratamento de escolha para estes tumores é a crioterapia com resultados satisfatórios, sendo a fotocoagulação e a vitrectomia reservadas para situações específicas ${ }^{(2,7-8)}$. No estudo retrospectivo supracitado ${ }^{(7)}, 54$ pacientes $(42 \%)$ foram tratados com crioterapia, $7(5 \%)$ foram submetidos à fotocoagulação, sendo que os $53 \%$ restantes foram observados ou tratados com outras modalidades de terapia.

Autores descrevem o tratamento com crioterapia em 4 de 5 olhos estudados, obtendo regressão do tumor em todos os olhos $\operatorname{tratados}^{(2)}$.

No presente estudo, os tumores vasculares mostraram-se resistentes ao tratamento com fotocoagulação, sendo necessária a opção por vitrectomia no caso 1 e nova fotocoagulação no caso 2. Apesar da resistência inicial, as massas vasculares apresentaram regressão no término do tratamento com melhora da acuidade visual.

\section{CONCLUSÃO}

Conclui-se, portanto que, o tumor vasoproliferativo idiopático da retina apresenta características que o distinguem do hemangioma capilar da doença de Von-Hippel e deve ser lembrado no diagnóstico diferencial das hemorragias vítreas recidivantes. Ressalta-se ainda que, a fotocoagulação e a vitrectomia com aplicação de endolaser parecem ser viáveis no tratamento desta doença.

\begin{abstract}
Purpose: The authors describe two cases of a rare and illustrative presumed vasoproliferative tumor of the retina associated with recurring vitreous hemorrhage in two young and healthy patients. Method: Two eyes of two patients were examined by fundoscopy, retinography, fluorescein angiography and ultrasonography. Results: In both cases, the vascular tumor was unilateral, solitary and showed ultrasonographic evidence of vitreous attachment. The differential diagnosis of the disease and options for treatment with cryotherapy, photocoagulation and vitrectomy are discussed. Conclusion: The authors point out the importance of recognizing of idiopathic vasoproliferative tumor of the retina in cases of recidivant vitreous hemorrhage.
\end{abstract}

Keywords: Hemangioma/pathology; Retinal neoplasms/pathology; Retinal vessels/pathology; Neovascularization pathologic; Vitreous hemorrhage; Case report

\section{REFERÊNCIAS}

1. Shields JA, Decker WL, Sanborn GE. Presumed acquired retinal hemangiomas. Ophthalmology 1983;90:1292-300.

2. Campochiaro PA, Conway BP. Hemangioma-like masses of the retina. Arch Ophthalmol 1988;106:1409-13.

3. Welch RB. Von Hippel-Lindau disease: the recognition and treatment of early angiomatosis retinal and the use of cryosurgery as an adjunct to therapy. Trans Am Ophthalmol Soc 1970;68:367-424.

4. Barr CC, Rice TA, Michels RG: Angioma-like mass in a patient with retrolental fibroplasia. Am J Ophthalmol 1980;89:647-50.

5. Bonanomi MTBC. Neovascularização da retina em hemoglobinopatia SC e hemorragia vítrea. Arq Bras Oftalmol 1997;60:24-33.

6. Egerer I, Tasman W, Tomer TT. Coats disease. Arch Ophthalmol 1974;92: 109-12.

7. Shields CL, Shields JA, Barrett J, De Potter P. Vasoproliferative tumors of the ocular fundus. Classification and clinical manifestations in 103 patients. Arch Opthalmol 1995;113:615-23.

8. Jampol LM, Goldbaum MH. Peripheral proliferative retinopathies. Surv Ophthalmol $1980 ; 25: 1-14$

\title{
ABO ELETRÔNICO
}

\author{
Novo site
}

\section{Acesso: http://www.abonet.com.br}

